\title{
Equivalent to Represent Inertial and Primary Frequency Control Effects of an External System
}

Weckesser, Johannes Tilman Gabriel; Van Cutsem, Thierry

Published in:

I E T Generation, Transmission and Distribution

Link to article, DOI:

10.1049/iet-gtd.2016.1641

Publication date:

2017

Document Version

Peer reviewed version

Link back to DTU Orbit

Citation (APA):

Weckesser, J. T. G., \& Van Cutsem, T. (2017). Equivalent to Represent Inertial and Primary Frequency Control Effects of an External System. I E T Generation, Transmission and Distribution. https://doi.org/10.1049/ietgtd.2016.1641

\section{General rights}

Copyright and moral rights for the publications made accessible in the public portal are retained by the authors and/or other copyright owners and it is a condition of accessing publications that users recognise and abide by the legal requirements associated with these rights.

- Users may download and print one copy of any publication from the public portal for the purpose of private study or research.

- You may not further distribute the material or use it for any profit-making activity or commercial gain

- You may freely distribute the URL identifying the publication in the public portal 


\title{
An Equivalent to Represent Inertial and Primary Frequency Control Effects of an External System*
}

\author{
Tilman Weckesser ${ }^{1, \dagger}$ and Thierry Van Cutsem ${ }^{2}$ \\ ${ }^{1}$ Dept. Electrical Engineering \& Computer Science, University of Liège, Belgium. \\ ${ }^{2}$ Fund for Scientific Research (FNRS) at the University of Liège, Belgium. \\ †j.weckesser@ulg.ac.be
}

\begin{abstract}
This paper presents a novel equivalent, which is suitable for simulation of inertial and primary frequency control effects. In the model reduction procedure, dynamic power injectors are used to replace the external system and to mimic its dynamic behavior. The parameters of the equivalents are tuned with a simple approach presented in this paper. The effectiveness of the proposed method is demonstrated on a modified version of the ENTSO-E Dynamic Study Model. The results show that the system frequency response of the unreduced system is retained and a speedup of the simulations of around 4.0 is achieved.
\end{abstract}

\section{Introduction}

Today's power systems are increasing in size and complexity. Due to forces such as the globalization of electricity market, where an increasing share of power is traded across national and regional borders, individual (e.g. national) power systems have stronger interactions with their neighboring systems. This requires, when assessing stability of a particular system, that adjacent systems are represented with suitable dynamic models. However, it is constraining and impractical to carry out stability and security analyses on the full model, which includes detailed models of all neighboring power systems. These analyses are usually carried out with focus on a certain part of the system, called study system. The rest is referred to as external system. The size of the dynamic model to be considered in the analysis can be decreased by equivalencing the external system. This results in a reduced model comprising the study system and some equivalent. For this reason, the full model is also referred to as unreduced system. It is crucial that the equivalencing processes retain the effect of the external system on the study system.

Moreover, generally the study system is known in more detail and, hence, represented with more detailed models, while the models in the external system can be more generic. Inadequately tuned generic models can result in spurious model interactions or even instability in time-domain simulations, which are known not to occur in the real interconnected system.

In the past various methods for determining static and dynamic equivalents have been proposed. As described in [1], equivalencing methods can roughly be divided into two groups. The first group requires no knowledge about the configuration and parameters of the external system. Usually these methods solely utilize measurements from the study system and its boundary (for example

\footnotetext{
* This paper is a postprint of a paper submitted to and accepted for publication in IET Generation, Transmission \& Distribution and is subject to Institution of Engineering and Technology Copyright. The copy of record is available at the IET Digital Library
} 
those described in $[2,3])$. The second group requires knowledge about the external system and the methods are generally referred to as model reduction methods. The resulting equivalents can be suitable for static and/or dynamic studies.

One of the first developed static network equivalents is the so-called Ward equivalent, which was introduced in [4] and further developed in [5]. In the Ward equivalent, the generators and loads in the external system are converted into constant current injectors, which then allows elimination of all external buses. The authors of [6] presented an extension of the equivalent to enable steadystate assessment of generator outages. For that purpose, the effect of the speed governors of the generators in the external system were included in the derivation of the equivalent. In [7] the socalled generalized Ward equivalent was introduced. This extension allowed to account for changes in reactive power injection from the external system, by expressing it solely as a function of the bus voltages at the study system's boundary. A dynamic Ward equivalent for transient stability analysis was discussed in [8]. It allows to eliminate all buses in the external system where voltage dependent loads are connected.

Another approach uses REI (Radial, Equivalent and Independent) equivalents. The method was first presented in [9] and aimed at replacing a set of nodes by one new equivalent node. In order to perform the reduction, a zero loss network is setup, which connects all load (resp. generator) buses of the external system with a new fictitious bus. Subsequently, the generators and loads of the external system are merged at the new buses and the external nodes are eliminated. A dynamic REI equivalent suitable for transient stability studies was presented in [10]. The authors propose to determine REI equivalents at the boundary buses of the study system. At the newly created nodes, equivalent generators of coherent generator groups are defined. Then the machine parameters of the equivalent generators are determined through participation factors. For aggregation of coherent generators, several other approaches were developed. In [11] the parameters of an equivalent generator and its controllers are determined through a least-square fit of the transfer function of the coherent generator group. A trajectory sensitivity method is used in [12] to determine the exciter parameters of the aggregated generators. In [13], the authors propose a structure preserving technique to compute the parameters of the equivalent generator.

In this paper, a new method is proposed for representing the inertial and primary frequency control effects of an external system in time-domain simulation. It is assumed that the unreduced system is large and contains the study system. As outlined so far, most techniques for external system equivalencing involve merging the original synchronous machines into a number of equivalent machines. This raises at least two issues: (i) find proper parameters to assign to those fictitious machines (and their associated controllers) and (ii) avoid creating artificial modes of oscillations (not present in the unreduced model) involving those machines. Both issues are easily addressed with the model proposed in this paper. It consists of Dynamic Power Injectors (DPIs) distributed along the boundary of the study system and emulating the response of the external system to active power imbalance and frequency excursions. Note that the equivalent is not aimed at preserving interarea electromechanical oscillations, which are assumed to have proper damping. Instead, the equivalent focuses on the global mode (also referred to as common mode by some authors) of rotor oscillation, which is the well-damped oscillatory mode with the lowest frequency present in all machine speed responses. This mode is dominant in the evolution of the speed of the Center Of Inertia (COI) defined by:

$$
\omega_{C O I}^{\mathcal{W}}(t)=\frac{\sum_{i \in \mathcal{W}} \omega_{i}(t) \cdot M_{i}}{\sum_{i \in \mathcal{W}} M_{i}}
$$

where $\omega_{i}$ is the rotor speed of the $i$-th machine and $\mathcal{W}$ denotes the whole set of synchronous 
machines. The inertia constant $M_{i}$ of a machine $i$ is defined as.

$$
M_{i}=\frac{2 \cdot E_{k i n, i}}{S_{b a s e}}
$$

where $S_{b a s e}$ is the system $M V A$ base and $E_{k i n, i}$ the machine's kinetic energy at nominal speed.

To summarize, the overall goal of the proposed method is that both the reduced and unreduced systems expose the same COI speed response to a disturbance. For that purpose, the equivalent receives the $\mathrm{COI}$ speed of the reduced system as an input. Its parameters are tuned to emulate the external system inertial and primary frequency control effects.

The proposed equivalent increases the computational efficiency of repeated time-domain simulations, e.g. for the determination of secure operation limits and other parametric studies, or the simulation of numerous N-k contingencies. Of course another context of use is real-time dynamic security assessment.

The rest of the paper is structured as follows. In Section 2 the derivation of the dynamic power injector and the identification of its parameters are presented. Tests are reported in Section 3 using an modified version of the ENTSO-E Dynamic Study Model (DSM) [14]. The results evaluate the performance of the proposed novel equivalent. Finally, concluding remarks are offered in Section 4.

\section{Method}

\subsection{Objective}

The assumption is that a dynamic model for the entire unreduced power system is available. As mentioned above, the aim of the proposed method is to determine an equivalent of the external system such that the COI speed of the reduced system approaches with satisfactory accuracy the COI speed of the unreduced system subject to the same disturbance, i.e.

$$
\omega_{C O I, r e d}^{\mathcal{R}}(t) \simeq \omega_{C O I}^{\mathcal{W}}(t)
$$

with

$$
\omega_{\text {COI }, \text { red }}^{\mathcal{R}}(t)=\frac{\sum_{i \in \mathcal{R}} \omega_{i, r e d}(t) \cdot M_{i}}{\sum_{i \in \mathcal{R}} M_{i}}
$$

where $\mathcal{R}$ denotes the set of all synchronous machines in the reduced system and $\omega_{i, r e d}$ is the rotor speed of the $i$-th machine extracted from simulation of the reduced system.

This entails preserving the maximum deviation $\Delta \omega_{C O I, m}$, steady-state deviation $\Delta \omega_{C O I, s s}$, period of oscillation $T_{d}$ and settling time $t_{s}$ of the COI speed response, as shown in Fig. 1.

During the reduction process, it is generally of interest to retain a buffer zone around the study system. An abstract illustration of a study system surrounded by a buffer zone and the external system is depicted in Fig. 2a. The red $S-B$ tie lines are the branches connecting the study system to the buses in the buffer zone. Likewise, the black $B-E$ tie lines are the branches linking buses in the buffer zone to buses in the external system.

The purpose of the equivalent is to emulate the active power flows from the external system corresponding to the inertial and primary frequency control responses. For that purpose, it is proposed to place DPIs at the boundary buses of the buffer zone. In Fig. 2b the external system has been removed and the power flows through the $B-E$ tie lines have been replaced by the shown 


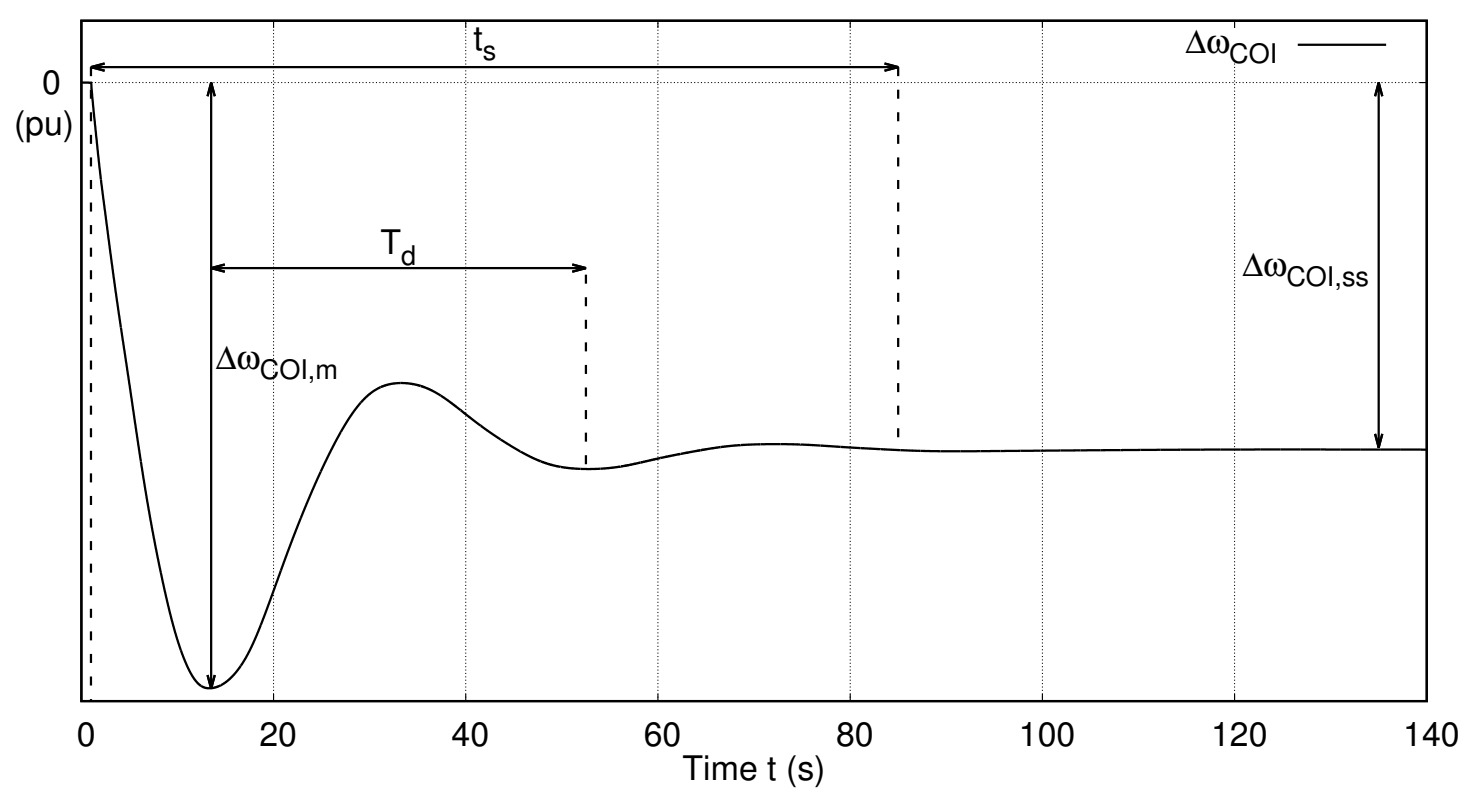

Fig. 1. Example of $\omega_{C O I}$ deviation following a disturbance.

DPIs. It should be noticed that at each boundary bus one DPI is placed irrespective of the number of connected $B-E$ tie lines. Consequently, the number of DPIs used to replace the external system corresponds to the number of boundary buses of the buffer zone.

\subsection{Modeling of the dynamic power injectors}

While the reactive power injection remains constant, the active power injection reacts to speed changes caused by disturbances in the study system. The block-diagram model of the proposed DPI is shown in Fig. 3. Ideally the input $\omega$ of the model should be $\omega_{C O I}^{\mathcal{W}}$ corresponding to the whole system. This is clearly not possible since, after reduction, the machines in the external system are no longer accessible. Instead it is proposed to use $\omega_{C O I, \text { red }}^{\mathcal{R}}$.

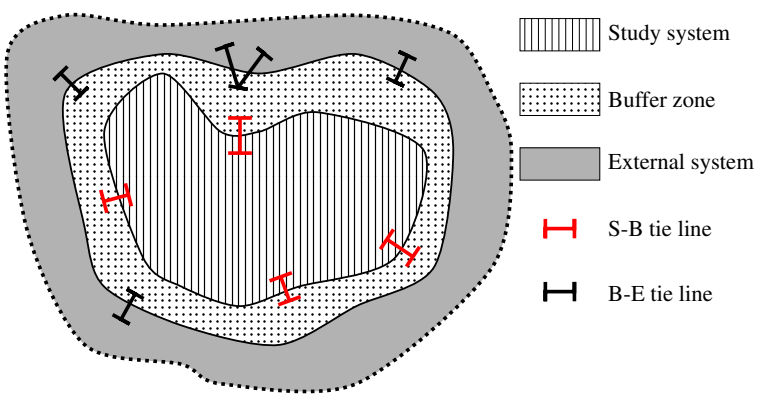

(a) Unreduced system divided into a study system, buffer zone and external system.

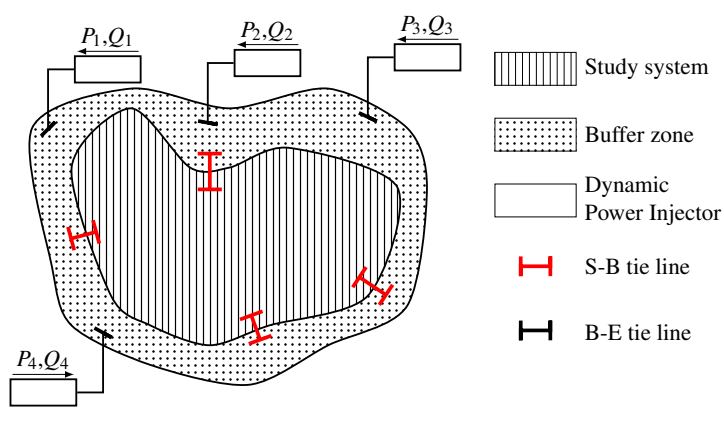

(b) Reduced system with external system replaced by DPIs.

Fig. 2. Abstract illustration of the reduction of a large power system model. 


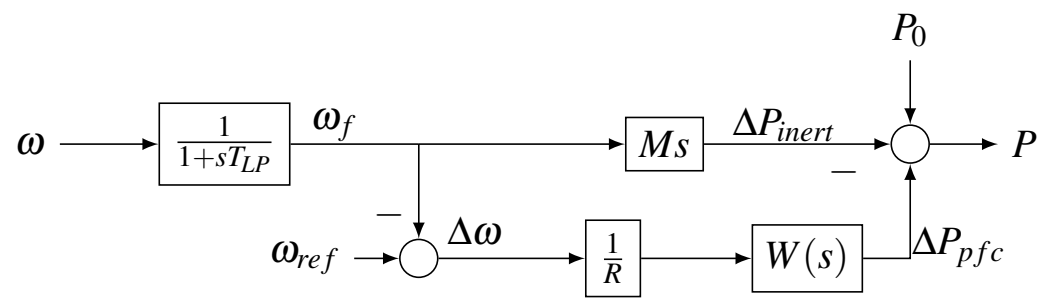

Fig. 3. Block diagram of dynamic power injector in pu.

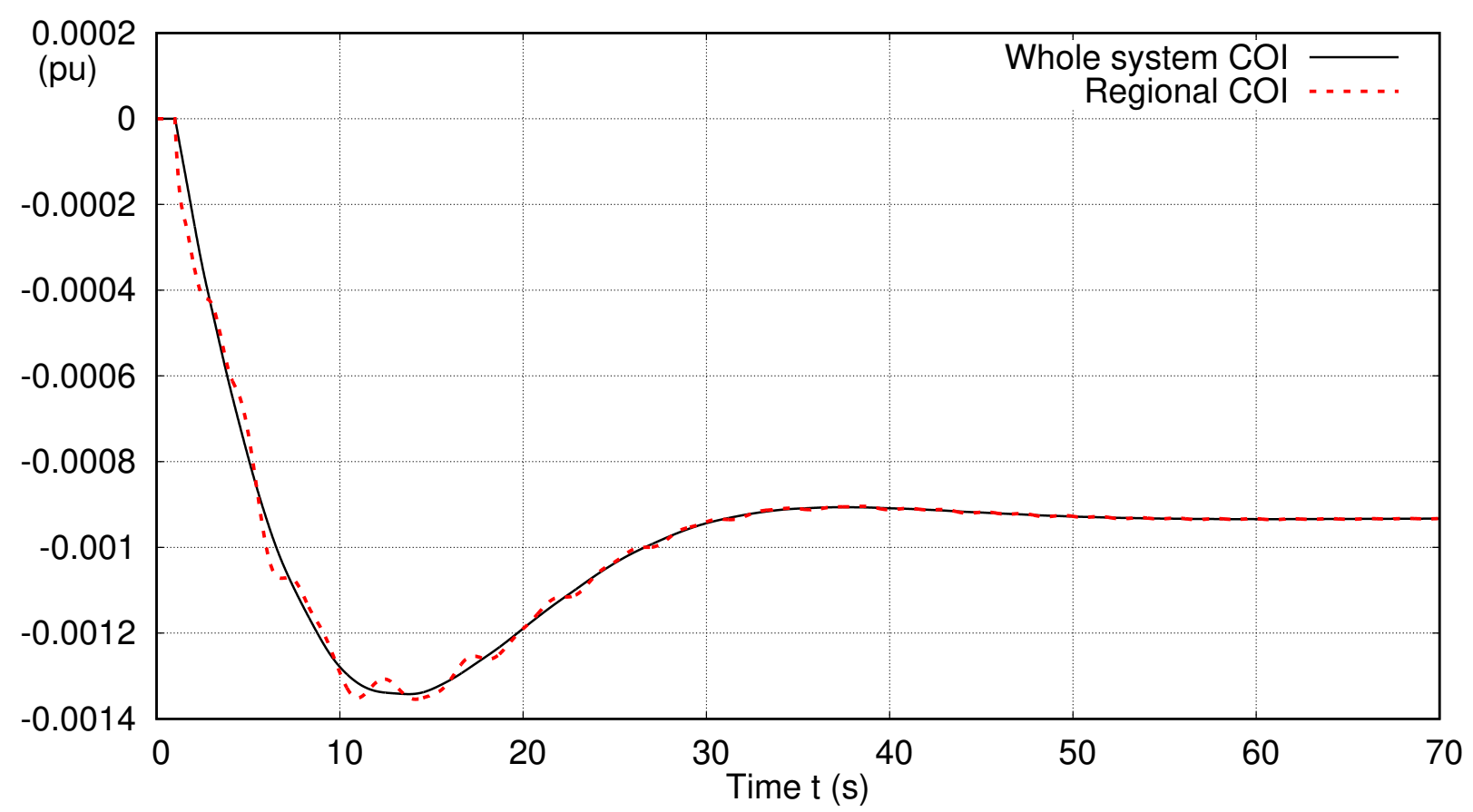

Fig. 4. Comparison of the speed deviation of the whole system $\left(\omega_{C O I}^{\mathcal{W}}\right)$ and of the study system $\left(\omega_{C O I, u n}^{\mathcal{R}}\right)$, both extracted from simulation of the unreduced system.

The validity of this choice is better illustrated through an example. The latter refers to the system detailed in Section 3, subject to the outage of a large generator at $t=1 \mathrm{~s}$. Figure 4 shows the time evolution of respectively: (i) the whole system COI speed obtained from (1) by summing over all machines in the unreduced system; (ii) the "regional" COI speed extracted from a simulation of the unreduced system:

$$
\omega_{C O I, \text { un }}^{\mathcal{R}}(t)=\frac{\sum_{i \in \mathcal{R}} \omega_{i}(t) \cdot M_{i}}{\sum_{i \in \mathcal{R}} M_{i}}
$$

where $\mathcal{R}$ again denotes the set of all machines retained in the reduced (study and buffer) system.

The regional COI exhibits an oscillation at a frequency of approximately $250 \mathrm{mHz}$ which corresponds to a known interarea mode of oscillation of the system of concern. When considering the speed of the whole system COI this oscillation is no longer visible. Indeed, by averaging over a larger number of rotor speeds, the resulting signal is better filtered. 


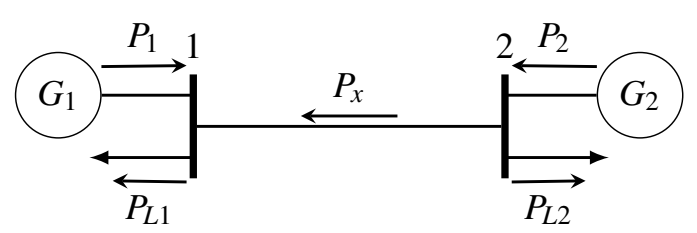

(a) Unreduced system

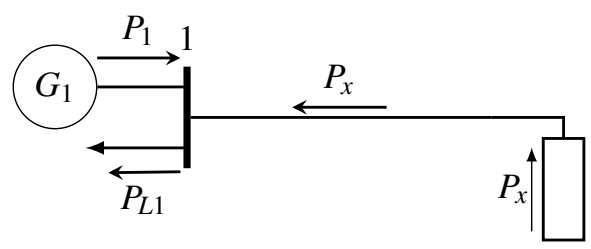

(b) Reduced system, where $G_{2}$ is replaced by a DPI

Fig. 5. Two-machine example

With $\omega_{C O I, r e d}^{\mathcal{R}}$ as input $\omega$, a simple low-pass filter is used, as shown in Fig. 3, to remove the effects of possible oscillations that the equivalent is not intended to reproduce. Optimally, the time constant $T_{L P}$ is chosen to cut-off the undesired higher frequency oscillations and better approach $\omega_{C O I}^{\mathcal{W}}$. The output of the block is the filtered speed $\omega_{f}$. The constant input $\omega_{\text {ref }}$ corresponds to a reference COI speed, which is equal to the COI speed in the initial system state and will be in most cases equal to $1.0 \mathrm{pu}$. In certain situations, e.g. when the initial state already corresponds to a deteriorated system condition, $\omega_{\text {ref }}$ may be different to $1.0 \mathrm{pu}$. The parameter $M$ corresponds to an equivalent inertia constant and $R$ is the speed droop. The transfer function $W(s)$ defines the dynamics of the primary frequency response of the injector. While the output $\Delta P_{\text {inert }}$ of the derivative block corresponds to the active power injection change due to preservation of the inertial effect, the output $\Delta P_{p f c}$ of the transfer function $W(s)$ emulates the primary frequency control contribution of the external system.

This model has the advantage, unlike equivalents using lumped machine models, of being less prone to additional oscillations, since the model does not include any rotor angle, but directly links active power injection to COI speed (similarly to an induction machine, whose torque is a function of speed).

Note that in theory, one machine located in the study system (or buffer zone) could be selected as reference and its rotor speed be used as input $\omega$ in the DPI model. As a single machine exhibits more higher-frequency (e.g. local) modes than the COI, a more elaborate low-pass filter should be considered. This is not needed when using $\omega_{C O I, \text { red }}^{\mathcal{R}}$, since the weighted average already contributes to filtering.

\subsection{Derivation on a simple two-machine example}

The derivation of the proposed DPI model is presented on basis of a simple lossless system with two machines $G_{1}$ and $G_{2}$, as shown in Fig. 5a. For sake of simplicity only the active power flow and injections are indicated. At bus 1 , generator $G_{1}$ produces active power $P_{1}$ and the load $L_{1}$ consumes $P_{L 1}$, likewise at bus 2 . The active power exchange between buses 1 and 2 is displayed as $P_{x}$. The active power balance of this lossless system can be expressed as:

$$
P_{1}=P_{L 1}-\underbrace{\left(P_{2}-P_{L 2}\right)}_{=P_{x}}
$$

In the following, the equations governing the machine dynamics are presented in the Laplace rather than the time domain. The swing equations of the machines are:

$$
\omega_{1}=\left(P_{m, 1}-P_{1}\right) \frac{1}{M_{1} s}
$$




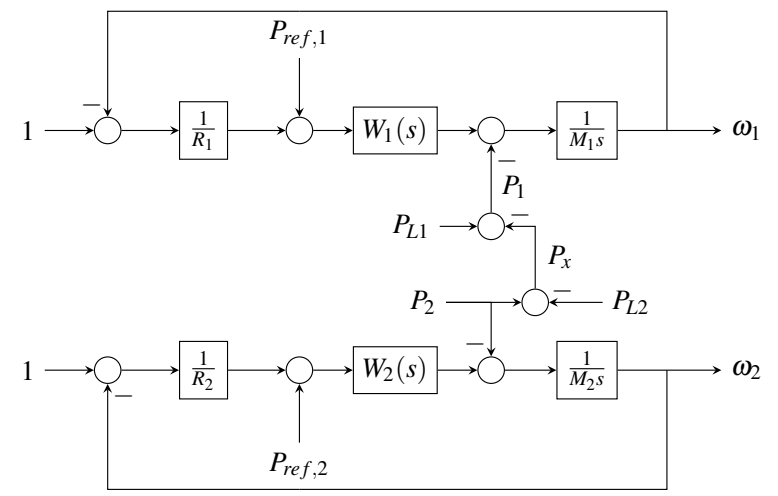

(a) Unreduced system

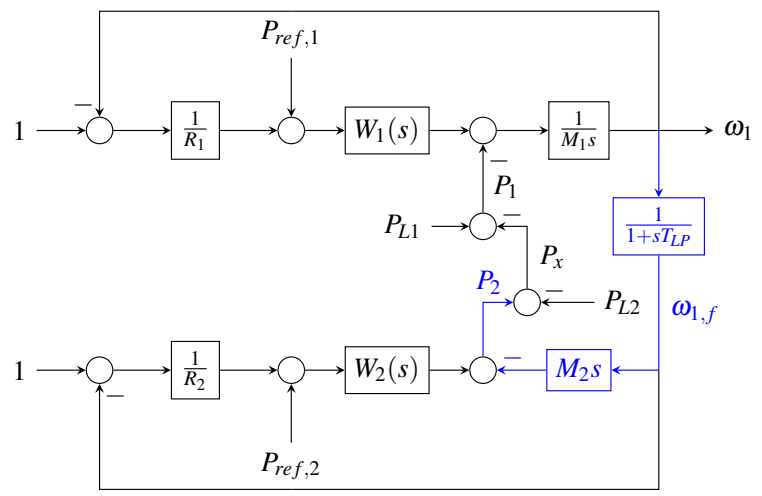

(b) Intermediate step during reduction

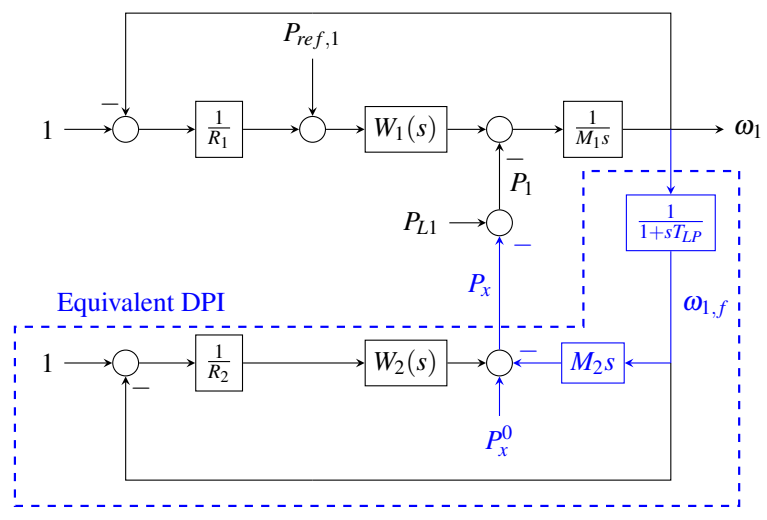

(c) Reduced system

Fig. 6. Block diagram of the two-machine system before, during and after reduction.

$$
\omega_{2}=\left(P_{m, 2}-P_{2}\right) \frac{1}{M_{2} s}
$$

where $P_{m, 1 / 2}$ are the mechanical powers and $M_{1,2}$ the respective inertia constants. Moreover, it is assumed that the machines are equipped with simple speed governors characterized by:

$$
\begin{aligned}
& P_{m, 1}=\left[P_{r e f, 1}+\left(1-\omega_{1}\right) \frac{1}{R_{1}}\right] W_{1}(s) \\
& P_{m, 2}=\left[P_{r e f, 2}+\left(1-\omega_{2}\right) \frac{1}{R_{2}}\right] W_{2}(s)
\end{aligned}
$$

$P_{r e f, 1 / 2}$ correspond to the dispatched active power set-points, $R_{1 / 2}$ are the speed droops and $W_{1 / 2}(s)$ are the transfer functions describing the turbine dynamics. Figure 6a shows the resulting block diagram of the two-machine system including a link corresponding to Eq.(6).

The steps that lead to replacing $G_{2}$ by a DPI (see Fig. 5b) are detailed next. The main assumption is that the active power response of machine $G_{2}$ is primarily influenced by $\omega_{C O I, \text { red }}^{\mathcal{R}}$, which in this simple example is merely $\omega_{1}$, the rotor speed of $G_{1}$. This assumption together with the application of the low-pass filter to $\omega_{1}$ yields:

$$
\omega_{2} \approx \omega_{1, f}=\frac{1}{1+s T_{L P}} \omega_{1}
$$


With (11) and by solving (8) for $P_{2}$, the active power of $G_{2}$ can be expressed as a function of $\omega_{1, f}$ :

$$
P_{2}=P_{m, 2}-M_{2} s \omega_{1, f}
$$

Moreover, with (11) the mechanical power (10) of $G_{2}$ can be expressed as a function of $\omega_{1, f}$, which can be inserted into (12):

$$
P_{2}=\left[P_{r e f, 2}+\left(1-\omega_{1, f}\right) \frac{1}{R_{2}}\right] W_{2}(s)-M_{2} s \omega_{1, f}
$$

This expression of $P_{2}$ as a function of $\omega_{1, f}$ is depicted in Fig. 6b. Using (6) and (13), the power exchange can be expressed as:

$$
P_{x}=P_{x}^{0}+\left(1-\omega_{1, f}\right) \frac{1}{R_{2}} W_{2}(s)-M_{2} s \omega_{1, f}
$$

with

$$
P_{x}^{0}=P_{r e f, 2} W_{2}(s)-P_{L 2}
$$

Figure $6 \mathrm{c}$ shows the block diagram of the resulting reduced system. The subsystem determining the power exchange $P_{x}$ corresponds to the DPI block diagram in Fig. 2b, when choosing the parameters as follows:

$$
R=R_{2}, M=M_{2}, \text { and } P_{0}=P_{x}^{0}
$$

In the rest of the paper the following transfer function is assumed for $W(s)$ :

$$
W(s)=\frac{1+s T_{z}}{1+s T_{p}}
$$

where $T_{z}$ and $T_{p}$ are time constants adjusted as explained in Section 2.4. With this transfer function, the primary frequency control of the DPI corresponds to a very simplified steam turbine model (referred to as TGOV1, see [15]). If (17) was compared to the transfer function of a steam turbine, $T_{z} / T_{p}$ would correspond to the fraction of the power developed in the high pressure turbine stage and $T_{p}$ to the reheater time constant [15, Fig. 2-1]. Experience shows that this transfer function is sufficient for representing primary frequency control effects of an external system with power dominantly provided by steam turbines (as in this paper, see Section 3.1). If the generation in the external system uses other turbines, e.g. gas or hydro, or converters, e.g. from PVs or type- 4 wind turbines, it may be necessary to use a different transfer function.

\subsection{Tuning the parameters of dynamic power injectors}

In order to correctly emulate the external system, the parameters of the equivalents need to be tuned. In the following, a simple approach is presented to tune the parameters of all DPIs simultaneously. The result is one set of parameters, which is used for all injectors.

An initial guess for the inertia constant can be obtained by consideration of the aggregated kinetic energy $E_{k i n, e x}$ of all $k$ machines in the external system:

$$
E_{k i n, e x}=\sum_{i=1}^{k} \frac{M_{i}}{2} S_{n o m, i}
$$


where $S_{n o m, i}$ is the nominal apparent power of the $i$-th generator. For each of the $j$ injectors the initial value of $M$ is computed as:

$$
M=c_{k i n} \frac{2 \cdot E_{k i n, e x}}{j \cdot S_{b a s e}}
$$

where $c_{k i n}$ is a correction factor, identical for all injectors (initially $c_{k i n}=1.0$ ).

An initial guess for the droop $R$ can be obtained by requiring that the composite frequency response characteristic $\beta$ (also called stiffness) [16] of the unreduced system is retained.

The contribution of the machines of the external system to the system's stiffness $\beta_{e x}$ is given by:

$$
\beta_{\text {ex }}=\sum_{i=1}^{k} \frac{1}{f_{\text {nom }}} \frac{P_{\text {nom }, i}}{R_{i}}
$$

where $f_{\text {nom }}$ is the nominal system frequency, $P_{n o m, i}$ is the nominal active power and $R_{i}$ is the speed droop of the $i$-th generator. The droop of each injector can then be determined as:

$$
R=\frac{1}{c_{\beta}} \frac{j \cdot S_{\text {base }}}{\beta_{\text {ex }} \cdot f_{\text {nom }}}
$$

where $c_{\beta}$ is another correction factor also identical for all injectors.

The selection of an initial value of $T_{z}$ and $T_{p}$ may not be obvious. In [16] standard parameters for a tandem-compound single reheat turbine of a fossil fueled unit were provided. Those parameters correspond to $T_{z}=2.1 \mathrm{~s}$ and $T_{p}=7.0 \mathrm{~s}$. However, based on experience for DPIs a reasonable initial value can be chosen in the range of $0.0-5.0 \mathrm{~s}$ and $5.0-10.0 \mathrm{~s}$, respectively.

In order to assess the impact of the four tunable parameters, namely $c_{\beta}, c_{k i n}, T_{z}$ and $T_{p}$, a parameter study was carried out, where a single parameter was varied and the remaining three were kept constant. The assumed contingency was in all cases the loss of a large generator at $t=1 \mathrm{~s}$ in the study system detailed in Section 3.1. Figure 7 shows the resulting speed deviation of $\omega_{C O I, \text { red }}^{\mathcal{R}}$. The latter is computed over all synchronous machines in the study system and buffer zone, after replacing the external system by DPIs.

The simulation results, where $c_{\beta}$ was varied between 1.0 and 2.0 , are shown in Fig. 7a. It can be observed that the variation mainly affects the steady-state speed deviation $\Delta \omega_{C O I, s s}$ and an increase of $c_{\beta}$ decreases it. This is expected, since it directly changes the droop of the DPIs. Figure $7 \mathrm{~b}$ displays similar results, when the correction factor $c_{k i n}$ is varied. This correction factor alters the inertia constant of the DPIs and, consequently, it is expected that it impacts the rate of change of $\omega_{C O I, r e d}^{\mathcal{R}}$. In Fig. $7 \mathbf{b}$ it can be observed that an increase of $c_{k i n}$ decreases the steepness of the curve. Moreover, the nadir increases while the oscillation is better damped. In the unreduced system an increase of $c_{k i n}$ would be equivalent to an increase of the system's inertia. The results of the variation of the parameter $T_{z}$ are shown in Fig. 7c. It can be observed that this parameter mainly affects the amplitude of the oscillation and, hence, the damping of the latter. The variation results of the last parameter $T_{p}$ are presented in Fig. $7 \mathrm{~d}$. In a simple turbine-governor model, this time constant would be related to the speed of the turbine and governor. A larger value would correspond to a slower response and vice versa. The graph shows that $T_{p}$ mainly impacts the nadir, where a larger time constant results in a larger maximum deviation.

Based on these observations, a heuristic approach is proposed to tune the four parameters. For that purpose, multiple simulations of the reduced system need to be carried out, while the parameters of the DPIs are varied, and the resulting evolutions of $\omega_{C O I, \text { red }}^{\mathcal{R}}$ are compared against 


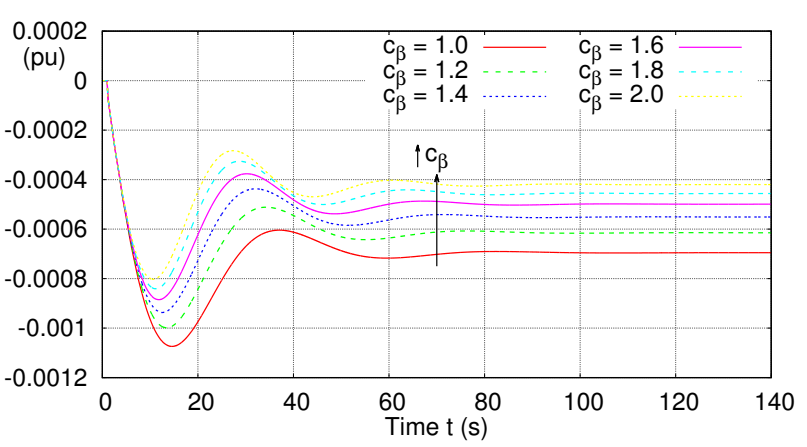

(a) Variation of $c_{\beta}$

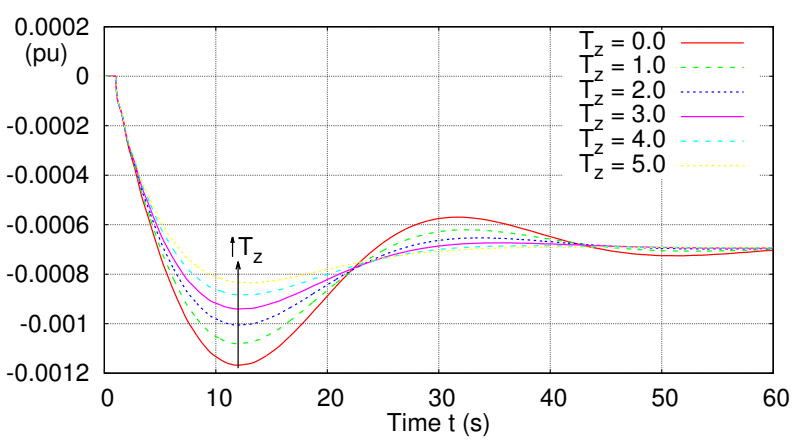

(c) Variation of $T_{z}$

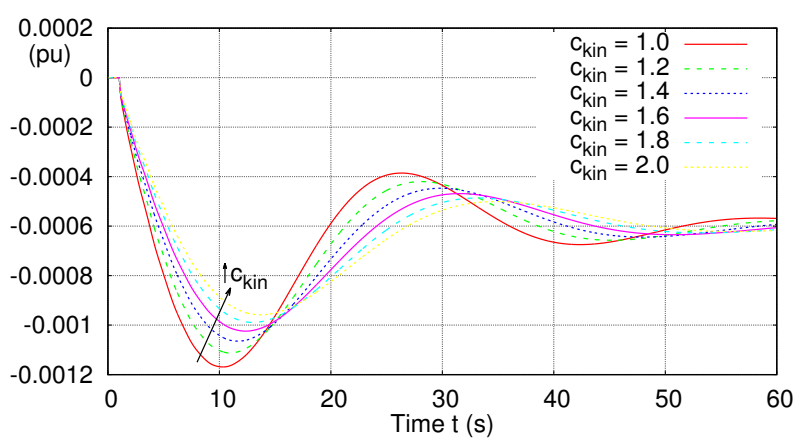

(b) Variation of $c_{k i n}$

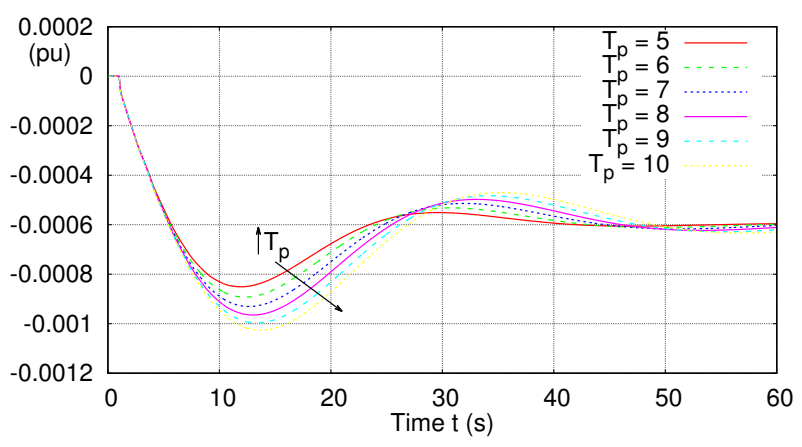

(d) Variation of $T_{p}$

Fig. 7. Demonstration of the impact of DPI parameters on the deviation of $\omega_{C O I, \text { red }}^{\mathcal{R}}$ in per unit following the loss of a large generator at $t=1 \mathrm{~s}$.

the corresponding evolution of $\omega_{C O I}^{\mathcal{W}}$ of the unreduced system. The steps are as follows (see also Fig. 1):

1. Adjust $c_{\beta}$ to fit $\Delta \omega_{C O I, s s}$ of the unreduced system (see Fig. 7a).

2. Tune $c_{k i n}$ to reach the rate of change and nadir $\Delta \omega_{C O I, m}$ of the COI speed in the unreduced system (see Fig. 7b).

3. Adjust $T_{p}$ and $T_{z}$ to fit $\Delta \omega_{C O I, m}, T_{d}$ and $t_{s}$ of the unreduced system (see Fig. 7c and Fig. 7d). Note: Re-adjusting of $c_{k i n}$ may be necessary.

\section{Results}

\subsection{Test system, simulation tool and scenario}

The results presented in this section relate to a modified version of the ENTSO-E DSM, which was originally presented in [14]. The study system was chosen to be Germany, some parts of which were represented in greater details for long-term dynamics studies. The model comprises approximately 27000 buses, 35000 branches and 900 generators.

The controllers of the generators in the ENTSO-E DSM have simplified dynamic models (see [14]). In some parts of the study system more detailed controller models were used. All loads are represented as $100 \%$ constant current and $100 \%$ constant admittance for the active and reactive power components, respectively. 
Table 1 Tuned parameter of DPIs.

\begin{tabular}{c|c|c|c|c|c|c}
$T_{L P}[\mathrm{~s}]$ & $c_{k i n}$ & $M[\mathrm{~s}]$ & $c_{\beta}$ & $R$ & $T_{z}[\mathrm{~s}]$ & $T_{p}[\mathrm{~s}]$ \\
\hline 0.622 & 1.45 & 186.8 & 1.02 & 0.024 & 2.5 & 9.8
\end{tabular}

The initial operating point is a scenario with high demand, high wind infeed, no PV infeed and low availability of conventional power plants in the study system. A buffer zone of 670 buses surrounds the study system, which leads to around 7000 buses, 9700 branches, 190 synchronous machines and 227 boundary buses, where DPIs are connected to replace the external system.

The simulation results have been obtained with the RAMSES software developed at the University of Liège [17]. The solver implements the technique presented in [18], which uses the COI of the previous discrete time as a reference for phasors. Additionally to the advantages of using the COI as reference, the COI computed at the last discrete time is an explicit (known) value and not a variable, which avoids the introduction of a dense row and column in the Jacobian used by the numerical solver.

Scenario 1: The assumed disturbance is the loss of a generator in the study system producing approx. $1200 \mathrm{MW}$. The resulting deviation of $\omega_{C O I}^{\mathcal{W}}$ in per unit can be seen in Fig. 4. A sharp decline can be observed, which reached a minimum of $-1.34 \cdot 10^{-3} \mathrm{pu}$ and settled at a steadystate speed deviation of $-0.93 \cdot 10^{-3} \mathrm{pu}$. This scenario has been used to tune the parameters of the DPIs.

Scenario 2: $\quad$ A second scenario, where another generator producing $840 \mathrm{MW}$ is tripped in the study system, is used for additional testing of the proposed equivalent.

\subsection{Parametrization of equivalent injectors}

The active and reactive powers of the DPIs are initialized to the power flow from the external system into the buffer zone at the respective boundary bus. The reactive power remains constant during the time simulation. In order to determine the time constant $T_{L P}$ of the low-pass filter, the period of the $\omega_{C O I}^{\mathcal{W}}$ response is considered. In Fig. 4 an oscillation period $T_{d}$ of approximately 39.0 s can be observed, which corresponds to a frequency of $25.6 \mathrm{mHz}$. In order to ensure that the low-pass filter does only filter oscillations with higher frequency, the cutoff frequency should be chosen at least one order of magnitude greater than $25.6 \mathrm{mHz}$, i.e. it should not be chosen lower than $256 \mathrm{mHz}$ in the test case.

Analysis of the Central European power system [19] and the Continental European power system [20] have shown that interarea oscillations of $250 \mathrm{mHz}$ may occur. For that reason, the cutoff frequency was chosen equal to the prior mentioned minimum value of $256 \mathrm{mHz}$, which corresponds to setting the time-constant $T_{L P}$ to $0.622 \mathrm{~s}$.

In order to determine the parameters $R, M, T_{z}$ and $T_{p}$, the approach presented in Section 2.4 was applied and the resulting parameter set is shown in Table 1.

\subsection{Speed of $\mathrm{COI}$ and individual machines}

As stated in Section 2, the objective of the proposed equivalent is to reproduce the inertial and speed governor response of the unreduced system. To this purpose, the COI speeds $\omega_{C O I}^{\mathcal{W}}$ and $\omega_{C O I, \text { red }}^{\mathcal{R}}$ are compared. It should be noted that $\omega_{C O I, \text { red }}^{\mathcal{R}}$ only involves the machines in the study 
system and buffer zone. The DPIs are not involved in the COI computation (but contribute to frequency support through the modulation of their active power).

Scenario 1: Figure 8a shows the above mentioned comparison. It can be observed that both COI speed evolutions coincide. Consequently, through tuning of the DPI parameters, the maximum deviation, steady-state deviation, oscillation period and settling time of the COI speed can be retained in the reduced system.

Figure $8 \mathrm{~b}$ shows the rotor speed of three individual machines in the study system. The solid lines corresponds to the speed evolution in the simulation of the unreduced system, while the dashed lines show the results from simulating the reduced system. During the transient period larger deviations can be observed, which is acceptable since the focus is on reproducing the global mode and long-term behavior. After the initial deviations, the speed evolutions of the three machines coincide in the two simulations, apart from higher frequency oscillations, which are filtered out as expected.

Scenario 2: $\quad$ Figure 9 shows the comparison of the COI speeds in the unreduced and the reduced system, when another generator is tripped at $t=1.0 \mathrm{~s}$. After the disturbance, in the simulation of the unreduced system the COI speed deviation drops to a minimum of $-9.45 \cdot 10^{-4} \mathrm{pu}$ and, eventually, settles at $-6.56 \cdot 10^{-4} \mathrm{pu}$. It can be observed that the evolution of the COI speed deviation in both simulations coincide, which confirms the accuracy of the proposed equivalent.

\subsection{Simulation speedup}

A major motivation for replacing an external system with an equivalent is that the system size reduction can lead to a significant simulation speedup. Figure 10 shows a comparison of the total elapsed time, when the contingency of Scenario 1 was simulated for $150.0 \mathrm{~s}$. Since the RAMSES software exploits parallelization [17], a comparison of the runtime as a function of the number of used cores is also presented. The simulation of the unreduced system takes $425.7 \mathrm{~s}$ using only one CPU core and decreases to $247.2 \mathrm{~s}$ when using four CPU cores. In the reduced system, the simulation requires between $104.6 \mathrm{~s}$ (single core) and $55.8 \mathrm{~s}$ (four cores). Hence, the speedup due to equivalencing of the external system is between 4.1 (single core) and 4.4 (four cores). The speedup obtained by both techniques combined is 7.6.

\section{Conclusion}

In this paper a novel equivalent was presented, which is suitable for simulation of inertial and primary frequency control effects. After reduction of a large power system, the components in the study system are modeled in detail, while the external system is replaced by DPIs attached at the periphery of the buffer zone. The DPIs emulate the power flow into the buffer zone. With a simple approach, the parameters of these injectors are tuned to mimic the response of the external system.

The proposed method was tested on a modified version of the ENTSO-E Dynamic Study Model. The results demonstrated that after tuning of the equivalent DPIs the COI speed response of the reduced system and the unreduced system coincide.

The model reduction allows achieving a computational speedup of around 4.0.

The equivalent may prove even more useful in future systems with progressively decreasing inertia, leading to larger frequency deviations. Looking further into the future, once synthetic 


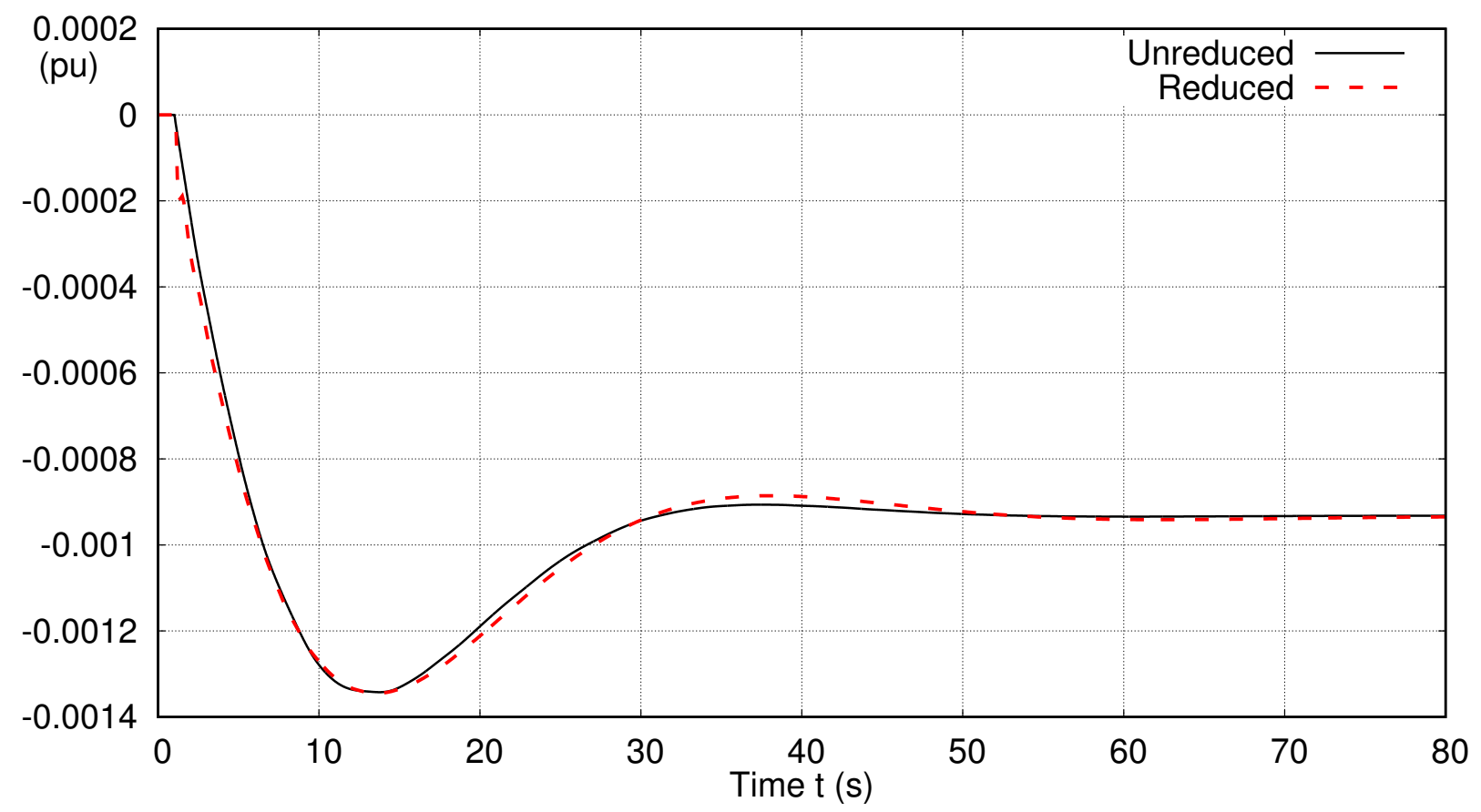

(a) Speed of COI in the unreduced and reduced system.

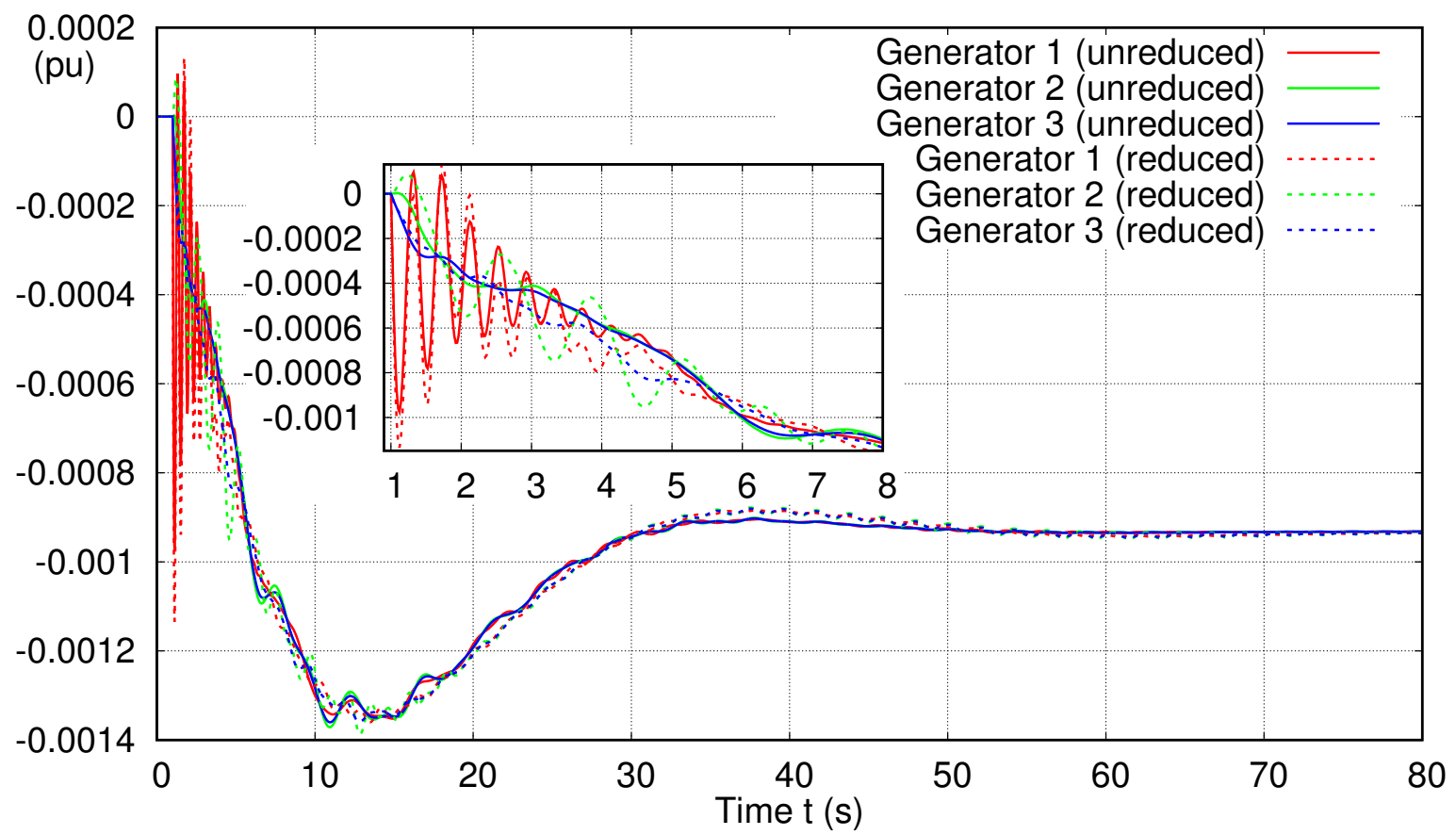

(b) Rotor speed of individual synchronous generators located in the study system with an embedded zoom on the transient period.

Fig. 8. Scenario 1 - Comparison of results from simulations of the unreduced and reduced system. 


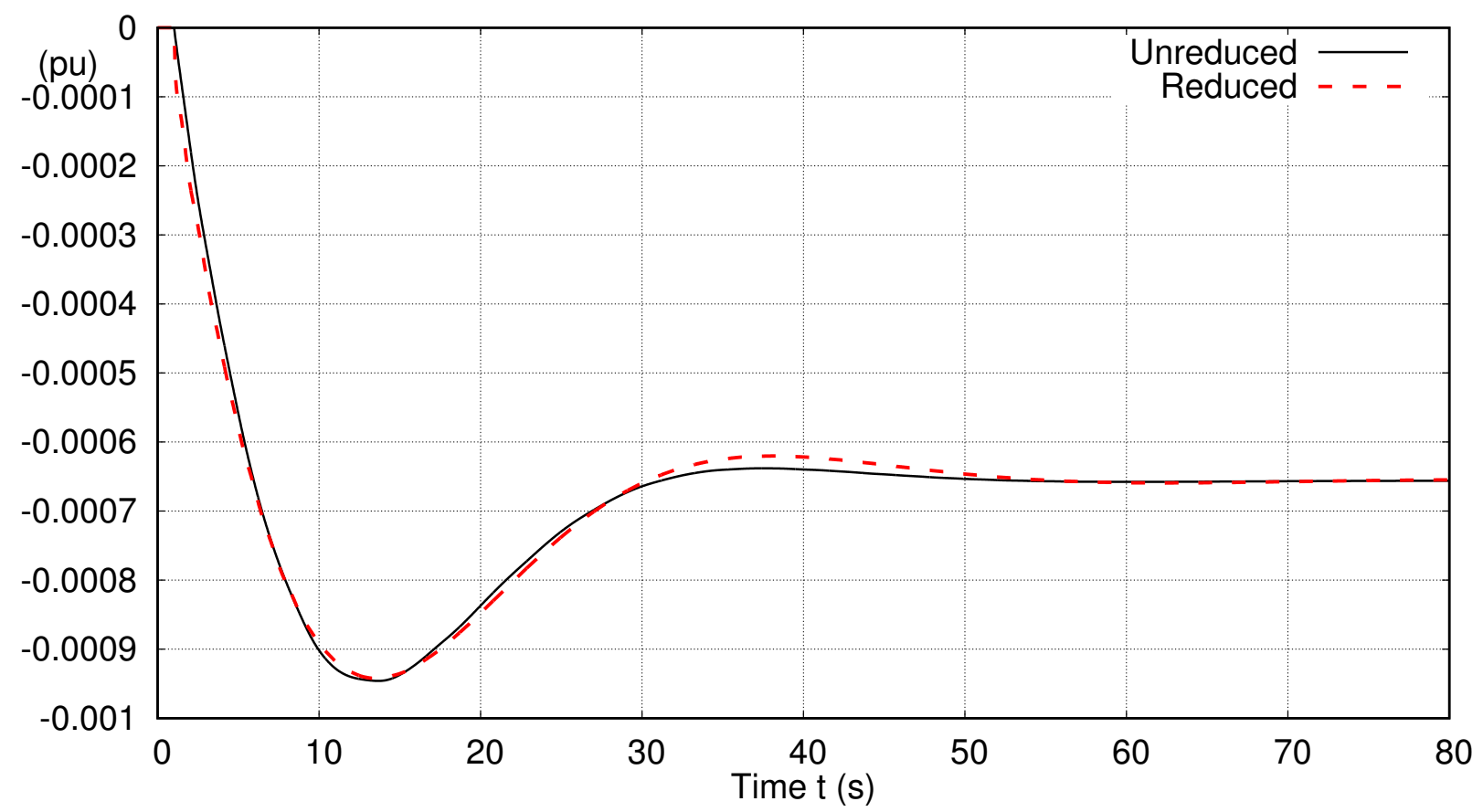

Fig. 9. Scenario 2 - Comparison of the speed of COI in the unreduced and reduced system.

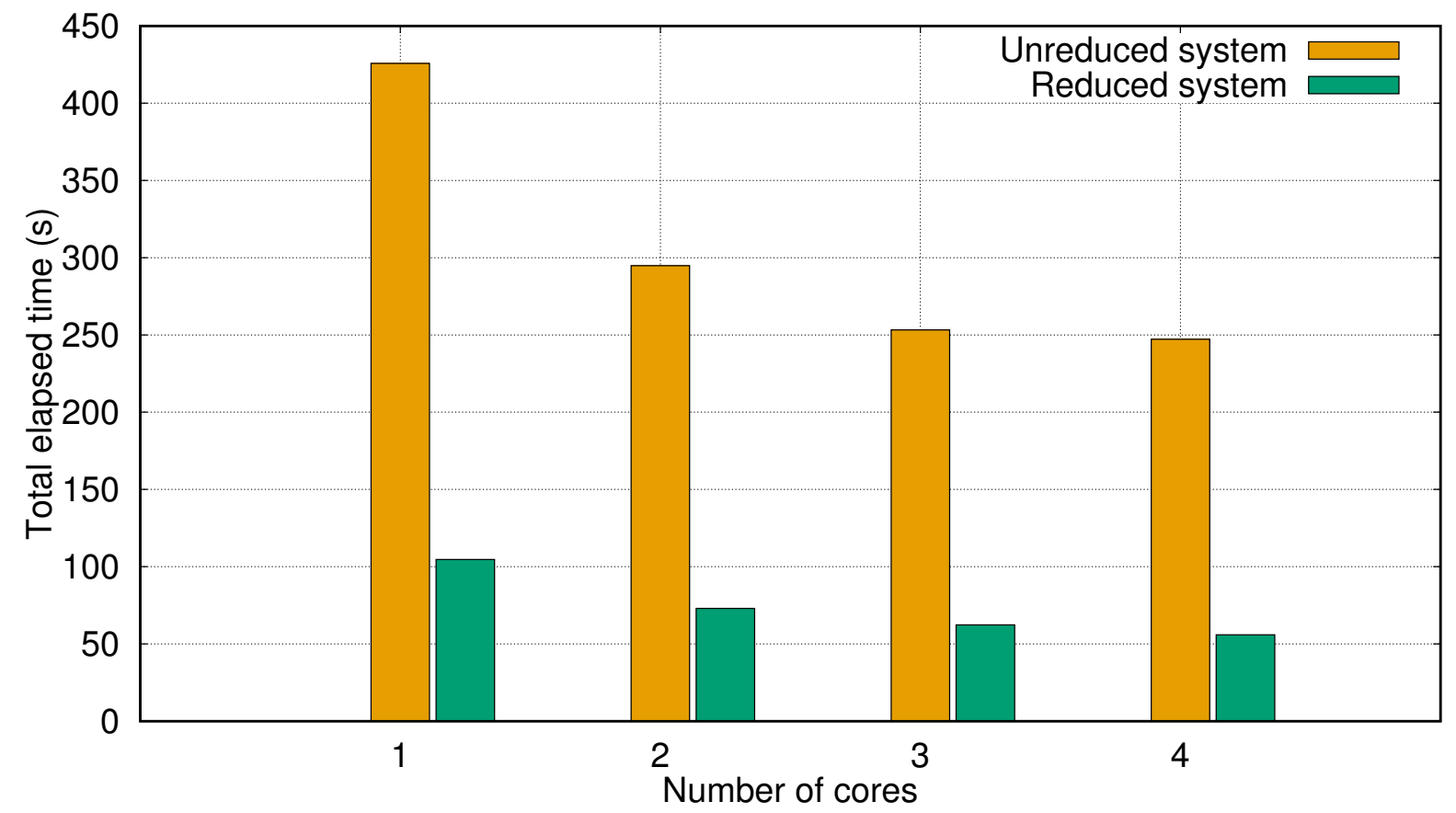

Fig. 10. Run time comparison of simulation of the unreduced and reduced system. 
inertia and frequency control will be taken over by converter interfaced generators, the equivalent will still be applicable, because it does not refer to synchronous machines specifically.

In order to ensure that the power flows into the study system are not distorted through the distributed DPIs with identical parameters, the latter could be tuned individually and/or several injectors could be clustered. This will be further investigated in the future.

\section{Acknowledgment}

This research was supported by the Amprion GmbH, Germany. We thank Dr. Eckhard Grebe, Franz Valeri, Klaus Vennemann and Dr. Roland Becker for their assistance in setting up the dynamic model as well as their expert insights in discussions.

\section{References}

[1] J. Machowski, J. W. Bialek, and J. R. Bumby, Power System Dynamics: Stability and Control, 2nd ed. John Wiley \& Sons, 2009.

[2] J. Dopazo, M. Dwarakanath, J. Li, and A. Sasson, "An external system equivalent model using real-time measurements for system security evaluation," IEEE Transactions on Power Apparatus and Systems, vol. 96, no. 2, pp. 431-446, 1977.

[3] X. Feng, Z. Lubosny, and J. W. Bialek, "Identification based dynamic equivalencing," in 2007 IEEE PowerTech Lausanne, July 2007, pp. 267-272.

[4] J. B. Ward, "Equivalent Circuits for Power-Flow Studies," AIEE Transactions, vol. 68, no. 1, pp. 373-382, 1949.

[5] A. Monticelli, S. Deckmann, A. Garcia, and B. Stott, "Real-time external equivalents for static security analysis," IEEE Transactions on Power Apparatus and Systems, vol. PAS-98, no. 2, pp. 498-508, 1979.

[6] F. Aschmoneit and J. Verstege, "An External System Equivalent for on-line Steady-State Generator Outage Simulation," IEEE Transactions on Power Apparatus and Systems, vol. PAS-98, no. 3, pp. 770-779, 1979.

[7] R. van Amerongen and H. van Meeteren, "A Generalised Ward Equivalent for Security Analysis," IEEE Transactions on Power Apparatus and Systems, vol. PAS-101, no. 6, pp. 15191526, jun 1982.

[8] T. Baldwin, L. Mili, and A. Phadke, "Dynamic Ward equivalents for transient stability analysis," IEEE Transactions on Power Systems, vol. 9, no. 1, pp. 59-67, feb 1994.

[9] P. Dimo, Nodal Analysis of Power Systems. Kent, England: Abacus Press, 1975.

[10] F. Milano and K. Srivastava, "Dynamic REI equivalents for short circuit and transient stability analyses," Electric Power Systems Research, vol. 79, no. 6, pp. 878-887, 2009.

[11] A. J. Germond and R. Podmore, "Dynamic Aggregation of Generating Unit Models," IEEE Transactions on Power Apparatus and Systems, vol. PAS-97, no. 4, pp. 1060-1069, 1978. 
[12] R. J. Galarza, J. H. Chow, W. W. Price, A. W. Hargrave, and P. M. Hirsch, "Aggregation of exciter models for constructing power system dynamic Equivalents," IEEE Transactions on Power Systems, vol. 13, no. 3, pp. 782-788, 1998.

[13] M. Ourari, L.-A. Dessaint, and V.-Q. Do, "Dynamic Equivalent Modeling of Large Power Systems Using Structure Preservation Technique," IEEE Transactions on Power Systems, vol. 21, no. 3, pp. 1284-1295, 2006.

[14] A. Semerow, S. Hohn, M. Luther, W. Sattinger, H. Abildgaard, A. D. Garcia, and G. Giannuzzi, "Dynamic Study Model for the interconnected power system of Continental Europe in different simulation tools," in 2015 IEEE Eindhoven PowerTech. IEEE, jun 2015, pp. 1-6.

[15] P. Pourbeik (Chair), "Dynamic models for turbine-governors in power system studies," IEEE Task Force on Turbine-Governor Modeling, Tech. Rep., 2013.

[16] P. Kundur, Power System Stability and Control, N. J. Balu and M. G. Lauby, Eds. McGrawHill Inc., 1994.

[17] P. Aristidou, D. Fabozzi, and T. Van Cutsem, "Dynamic simulation of large-scale power systems using a parallel schur-complement-based decomposition method," IEEE Transactions on Parallel and Distributed Systems, vol. 25, no. 10, pp. 2561-2570, Oct 2014.

[18] D. Fabozzi and T. Van Cutsem, "On angle references in long-term time-domain simulations," IEEE Transactions on Power Systems, vol. 26, no. 1, pp. 483-484, 2011.

[19] U. Bachmann, I. Erlich, and E. Grebe, "Analysis of interarea oscillations in the European electric power system in synchronous parallel operation with the Central-European networks," in 1999 IEEE PowerTech Budapest. IEEE, p. 49.

[20] ENTSO-E, “Analysis of CE Inter-Area Oscillations of 19 and 24 February 2011,” ENTSO-E, Brussels, Belgium, Tech. Rep., 2011. 